(2) TRANSCRIPTION

\section{Reaching a consensus}

p53 regulates gene transcription by binding to a consensus DNA sequence known as a response element (RE). Bei Wang, Ziwei Xiao and Ee Chee Ren have identified core elements in the p53 RE that help to define whether it activates or represses transcription.

The canonical p53 RE comprises two decamer motifs (RRRCWWGYYY, in which R is A or G, W is A or T and Y is C or T) separated by a spacer of $0-13$ base pairs. The $\mathrm{C}$ and $\mathrm{G}$ of the core CWWG motif are conserved, but variations occur in the WW motif, as well as in the flanking sequences, and are thought to affect the transcriptional behaviour of p53. Wang and colleagues compared the p53 REs present in the promoters of CDKN1A (which encodes p21 and which p53 activates) and LASP1, a gene that is repressed by $\mathrm{p} 53$. They used luciferase and other assays to analyse the transcriptional outcome of $\mathrm{p} 53$ binding to a series of CDKN1A and LASP1 mutant REs. Having characterized the p53 RE in $L A S P 1$, the authors established that the presence or absence of the spacer between the two decamer motifs had no effect on p53 transcriptional function. However, alterations to the dinucleotide core of the CWWG motif showed that if the core is AT, AA or TT then the $\mathrm{RE}$ is activating, whereas $\mathrm{CG}, \mathrm{GG}$, TG, CC, GC and CA all result in repression.
What about the function of the flanking RRR or YYY triplet sequences? The authors found that the activating function of an AT dinucleotide can be suppressed if the flanking nucleotides are changed and that the influence of the flanking nucleotide is greater the closer it is to the core CWWG motif. Importantly, the authors found that changing the flanking triplets in a repressor RE can alter the degree of repression but not reverse it, whereas specific triplet sequences can change an activator core to a repressive one.

Using this information they found that 20 of 162 published p53
REs were erroneously assigned as either activators or repressors and that the number of repressive REs is greater than previously thought. Overall, activating p53 REs generally conform to the canonical sequence, whereas repressive p53 REs are likely to have a repressive dinucleotide with flanking nucleotides adjacent to the CWWG core that do not conform to the canonical sequence. These findings should help to further characterize p53-regulated transcriptional networks and establish whether mutations in p53 REs contribute to tumorigenesis.

Nicola McCarthy

ORIGINAL RESEARCH PAPER Wang, B., Xiao, Z. $\S$ Ren, E. C. Redefining the $\mathrm{p} 53$ response element. Proc. Natl Acad. Sci. USA 13 Jul 2009 (doi:10.1073/pnas.0903284106)

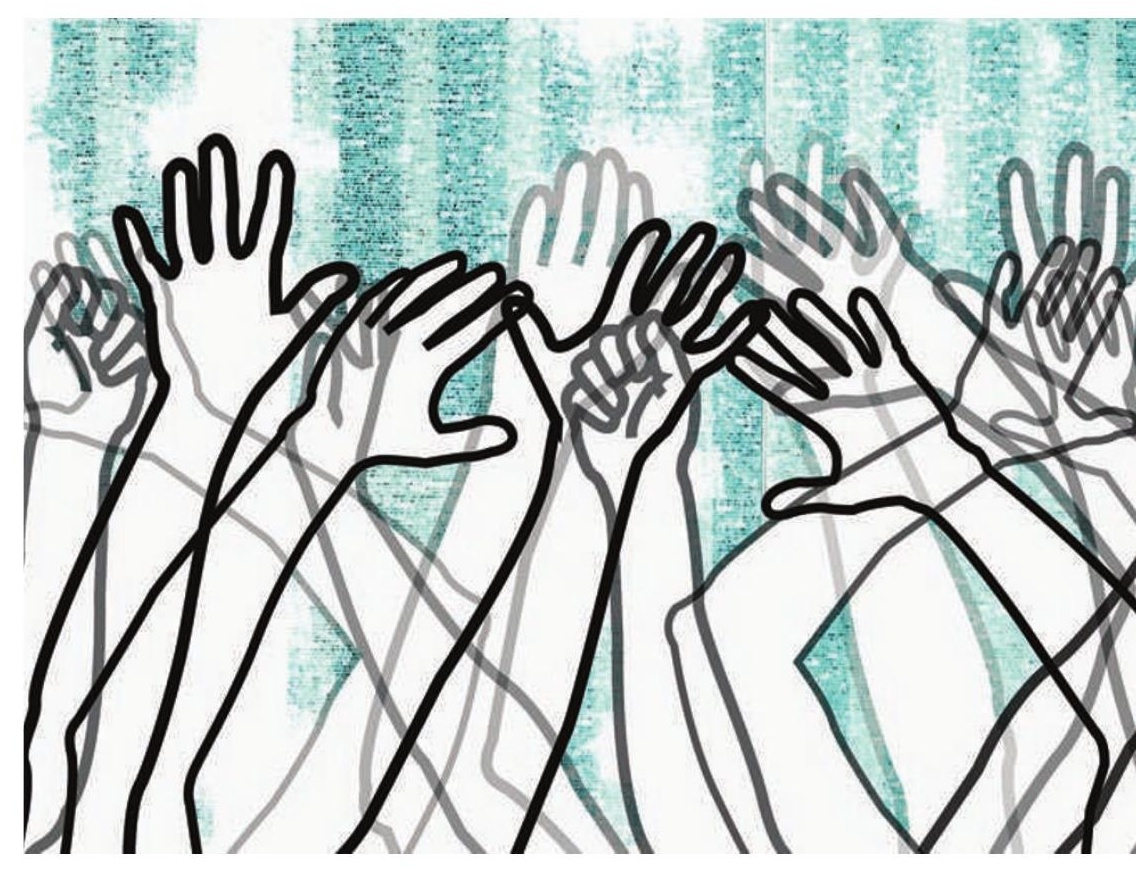

